\title{
Détermination de la diffusivité thermique et de la conductivité thermique de revêtements à haute température par la méthode flash
}

\section{Determination of the thermal diffusivity and thermal conductivity of coatings at high temperature by flash method}

\author{
Bruno HAY, Guillaume DAVEE, Jacques HAMEURY, Olivier ENOUF et Lydia RONGIONE
}

Laboratoire commun de métrologie LNE-Cnam (LCM)

Laboratoire national de métrologie et d'essais (LNE), 29 Avenue Roger Hennequin, 78197 Trappes Cedex, France, bruno.hay@lne.fr.

\section{Résumé}

Les revêtements céramiques épais déposés par projection plasma sont largement utilisés à haute température comme boucliers thermiques ou comme revêtements résistants à l'usure et à la corrosion. La caractérisation de la diffusivité thermique et de la conductivité thermique de ces matériaux est particulièrement importante pour les revêtements utilisés dans les domaines aéronautique, automobile et de production d'énergie. Il est donc nécessaire de disposer d'un équipement de référence pour la mesure de ces propriétés thermiques dans une large plage de températures et avec des incertitudes de mesure fiables, permettant d'effectuer des mesures et des étalonnages traçables au SI. Le diffusivimètre de référence du LNE, opérationnel depuis plusieurs années pour les mesures de la diffusivité thermique de matériaux homogènes moyens et bons conducteurs thermiques, fonctionne sur le principe de la méthode flash en face arrière et est relativement mal adapté aux mesures réalisées sur un revêtement (problèmes liés à l'épaisseur du substrat). L'installation de mesure existante a donc été modifiée, afin de permettre de déterminer la diffusivité thermique de revêtements à partir de la mesure de la température de la face soumise à l'impulsion (méthode flash avant) ou de la face opposée (méthode flash arrière). Elle permet par ailleurs, moyennant certaines conditions, de mesurer simultanément la diffusivité thermique et l'effusivité thermique de ces revêtements à haute température par la méthode flash «face avant ». Cette méthode a d'abord été validée, entre la température ambiante et $1400^{\circ} \mathrm{C}$, en comparant les résultats de mesure obtenus sur deux matériaux homogènes bien connus (fer Armco et graphite Poco) avec ceux obtenus en utilisant la méthode flash «face arrière ». Les valeurs de la diffusivité thermique ainsi obtenues ont un écart relatif inférieur à 3,5\%, dépendant de la température et des matériaux, avec une incertitude relative élargie inférieure à 5,5\%. La méthode flash «face avant » a ensuite été appliquée à la détermination de la conductivité thermique d'un revêtement d'oxyde de chrome déposé sur un substrat en alliage de fer, entre $23{ }^{\circ} \mathrm{C}$ et $800{ }^{\circ} \mathrm{C}$, en exploitant des mesures simultanées de la diffusivité thermique et de l'effusivité thermique. Les résultats ont été comparés aux valeurs de la conductivité thermique déterminées par une méthode indirecte à partir de la diffusivité thermique, de la capacité thermique massique et de la masse volumique. Les valeurs de la conductivité thermique mesurées en utilisant les deux méthodes sont en bon accord, avec un écart relatif inférieur à $7 \%$, et se situent dans la plage des incertitudes de mesure.

MOTS CLÉS : CONDUCTIVITÉ THERMIQUE, DIFFUSIVITÉ THERMIQUE, DÉPÔT CÉRAMIQUE, HAUTE TEMPÉRATURE, MÉTHODE FLASH, OXYDE DE CHROME.
Abstract
Thick ceramic coatings deposited by plasma spraying techniques are widely used as heat, wear and corrosion resistant coatings at high temperature. The characterization of the thermal diffusivity and the thermal conductivity of these materials is especially important for coat- ings used in aeronautics, automotive and power generation industries. It is therefore necessary to have a reference facility for the measurement of these thermal properties over a wide range of temperature with re- liable measurement uncertainties. LNE reference diffusivimeter, which is used for several years for measuring thermal diffusivity of homoge- neous materials, is based on the principle of the rear-face flash method. This method is not convenient for the study of coatings or multilayered materials. The existing LNE facility has been thus set-up in order to en- able the determination of the thermal diffusivity from the temperature of the front face or the rear face of the tested specimen. It also allows to measure simultaneously the thermal diffusivity and thermal effusivity of these coatings at high temperature by front-face flash method. This method has been first validated from room temperature to $1400{ }^{\circ} \mathrm{C}$ by comparing the obtained results of thermal diffusivity of two well- known homogeneous materials (Armco iron and Poco graphite) with 
those determined by using rear-face technique. The measured thermal diffusivity values have relative deviation of less than $3.5 \%$ depending on temperature and materials, with an expanded uncertainty less than 5.5\%. The front-face laser flash method has then been applied to the determination of thermal conductivity of chromium oxide coating deposited on iron alloy substrate from $23{ }^{\circ} \mathrm{C}$ to $800{ }^{\circ} \mathrm{C}$, by using simultaneous measurements of thermal diffusivity and thermal effusivity. The results were compared to thermal conductivity values determined by indirect method from thermal diffusivity, specific heat and density. The thermal conductivity values measured by using both methods are in good agreement, with a relative deviation of less than 7\%, and are within the range of uncertainty of measurement.

KEY WORDS: CERAMIC COATING, CHROMIUM OXIDE, HIGH TEMPERATURE, FLASH METHOD, THERMAL DIFFUSIVITY, THERMAL CONDUCTIVITY.

\section{Introduction}

Des revêtements sont souvent déposés sur des matériaux à des fins de protection ou d'amélioration des propriétés optiques ou mécaniques de surface (durcissement par exemple). Un cas typique concerne les revêtements céramiques déposés par projection plasma sur des substrats métalliques, qui sont notamment utilisés dans l'aéronautique, l'automobile et les industries de production d'énergie comme bouclier thermique ou anti-usure à haute température. Dans les turbines à gaz, par exemple, les pannes matérielles critiques et les problèmes de détérioration résultent principalement d'attaques chimiques, de chocs thermiques et de phénomènes d'érosion. Des revêtements céramiques spécifiques ont donc été développés pour améliorer la résistance à la corrosion et à l'abrasion des composants métalliques, permettant ainsi de prolonger la durée de vie des turbines à gaz $[1,2]$. Par ailleurs, des céramiques de faible conductivité thermique (généralement des oxydes de zircone stabilisés à l'yttrium) sont utilisées comme barrières thermiques dans les moteurs diesel [3], les tuyères de fusées ou les pales de turbine [4]. Elles accroissent notamment la fiabilité et les performances des moteurs, et contribuent à réduire la consommation de carburant et les émissions de particules et de fumées [5]. Enfin, des revêtements céramiques (oxyde d'yttrium et phosphate de Lanthane notamment) sont également utilisés par l'industrie nucléaire pour la manipulation et le confinement de l'uranium fondu et d'autres matériaux réactifs [6].

Pour toutes ces applications, la durabilité de ces revêtements soumis à des cyclages thermiques répétés à haute température est une préoccupation majeure. Comme les matériaux sont susceptibles d'évoluer lors d'une utilisation à température élevée (par exemple par frittage), il est nécessaire d'estimer leurs propriétés thermophysiques dans des conditions de température similaires à celles rencontrées dans des applications réelles, afin de prédire leur comportement thermique. La morphologie d'un revêtement, sa masse volumique et sa conductivité thermique en particulier dépendent fortement du processus de déposition utilisé lors de sa mise en œuvre. Il est donc rarement possible d'estimer les propriétés thermophysiques d'un revêtement à partir de celles d'un matériau massif de même composition. Pour éviter un délaminage « destructif» entre le substrat et le revêtement, qui entraînerait inévitablement une modification de la structure de ce dernier, les mesures des propriétés thermiques doivent être effectuées directement sur le composite multicouche. Pour cela, le diffusivimètre du LNE a été modifié pour permettre d'étudier les matériaux bicouches par méthodes « flash » en face arrière et en face avant, et d'évaluer ainsi la diffusivité thermique $(a)$ et la conductivité thermique $(\lambda)$ de tels revêtements à haute température avec une incertitude maîtrisée. L'objectif général de cette approche est de disposer d'une installation de référence permettant de réaliser des mesures de propriétés thermiques de ce type de matériaux qui soient fiables et traçables d'un point de vue métrologique. Cet article décrit la méthode de mesure mise en œuvre, la conception et l'optimisation de l'appareillage ainsi que sa validation métrologique. Les résultats obtenus sur une couche d'oxyde de chrome déposée sur un substrat en alliage de fer sont présentés et commentés.

\section{Principe de mesure}

Le LNE effectue depuis de nombreuses années des mesures de diffusivité thermique sur des matériaux homogènes jusqu'à $1400^{\circ} \mathrm{C}$, sous vide ou sous atmosphère neutre, en utilisant une installation de référence [7] basée sur le principe «traditionnel» de la méthode «flash laser » en face arrière [8]. Un échantillon cylindrique est soumis sur sa face avant à une impulsion laser de courte durée, et le signal transitoire de température induit sur sa face arrière est mesuré en fonction du temps. Dans le cas d'un matériau homogène massif, la diffusivité thermique est estimée selon la méthode "des moments temporels partiels » [9]. Cette méthode d'estimation, qui a notamment été utilisée par le LNE dans le processus de certification du Pyroceram 9606 comme matériau de référence (BCR-724) [10], n'est pas adaptée pour l'étude des revêtements ou des matériaux multicouches.

Une procédure d'estimation permettant l'évaluation de la diffusivité thermique de tels dépôts par les méthodes « flash laser » face arrière et face avant avec l'installation du LNE a été proposée par Krapez [11,12]. Elle repose sur la minimisation de la différence entre la courbe expérimentale «température en fonction du temps » (thermogramme) et les valeurs théoriques correspondantes, obtenues par modélisation du transfert de chaleur dans un système multicouche. L'équation de conduction de la chaleur est résolue analytiquement dans l'espace de Laplace par la méthode des quadripôles [13], qui permet notamment d'exprimer de façon concise la température d'un matériau multicouche en régime dynamique. Un algorithme de Stehfest [14] pour l'inversion numérique des transformées de Laplace est appliqué aux résultats afin d'obtenir le signal transitoire de température des faces avant ou arrière de l'éprouvette. Les propriétés thermophysiques du substrat étant connues, l'estimation de la diffusivité thermique du revêtement est effectuée en utilisant une méthode des moindres carrés. Une étude de la sensibilité du modèle théorique utilisé a été réalisée afin de définir les limites du domaine temporel d'analyse conduisant aux meilleurs résultats en termes 


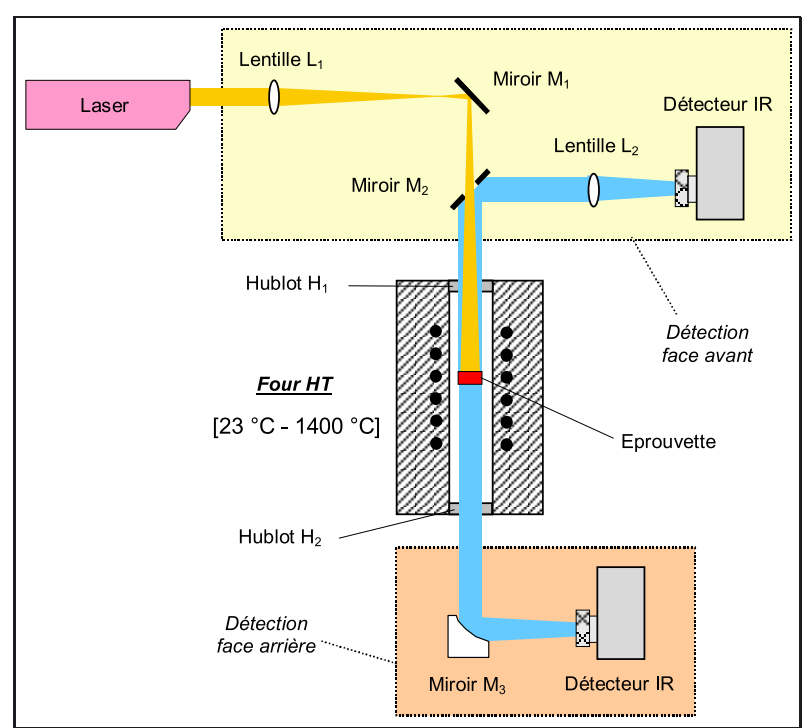

Fig. 1. - Schéma de principe du diffusivimètre du LNE présentant les systèmes de détection en faces avant et arrière de l'éprouvette.

de fiabilité et d'incertitude des paramètres estimés (diffusivité thermique et pertes thermiques) [11,12]. Elle montre notamment que pour la méthode flash «face arrière », la sensibilité diminue lorsque l'épaisseur du substrat augmente, en raison du retard et de l'atténuation du signal thermique initial naturellement introduits par le substrat. Dans le cas d'échantillons épais, il est alors préférable d'appliquer la méthode «flash » face avant qui consiste à déterminer la diffusivité thermique du revêtement à partir de l'analyse de la décroissance de la température de la face avant de l'éprouvette. Par ailleurs, pour autant qu'il existe une différence significative entre les propriétés thermiques du revêtement et du substrat, cette méthode permet de mesurer simultanément la diffusivité thermique $(a)$ et l'effusivité thermique $(e)$ d'un revêtement quelle que soit l'épaisseur du substrat. La conductivité thermique du revêtement est calculée à partir de ces deux grandeurs d'après la relation (1).

$$
\lambda=e \cdot \sqrt{a}
$$

\section{Description du diffusivimètre du LNE}

Un schéma de l'installation de mesure du LNE présentant les systèmes de détections en faces avant et arrière de l'éprouvette est présenté sur la figure 1.

\subsection{Mesure en face arrière}

La description technique de l'excitation thermique de l'éprouvette, de la détection optique de sa température et de l'acquisition des données est rappelée ici pour l'installation initiale, c'est-à-dire pour la mesure par la méthode «flash » en face arrière.
Une excitation thermique de courte durée (environ $450 \mu \mathrm{s})$ est générée par un laser $\mathrm{Nd}$ :phosphate fonctionnant à la longueur d'onde de $1054 \mathrm{~nm}$. Le faisceau laser est dirigé par un miroir diélectrique plan $\mathrm{M}_{1}$ vers l'intérieur d'un four résistif permettant de chauffer une éprouvette ( $10 \mathrm{~mm}$ de diamètre et $1 \mathrm{~mm}$ à $5 \mathrm{~mm}$ d'épaisseur) jusqu'à $1400{ }^{\circ} \mathrm{C}$. Le four est un cylindre vertical fermé au deux extrémités par deux fenêtres en ZnSe, qui sont transparentes à la longueur d'onde du laser et sur la plage des longueurs d'onde de travail des détecteurs infrarouge utilisés. La température de l'échantillon est mesurée par un thermocouple de type $\mathrm{S}$ situé dans le porteéchantillon. Une photodiode est utilisée pour déterminer la durée et la forme de l'impulsion, ainsi que l'origine des temps qui correspond au moment où le faisceau laser irradie l'échantillon. L'élévation de la température de sa face arrière est mesurée au moyen de deux détecteurs infrarouges ( $\mathrm{HgCdTe}$ ou InSb en fonction de la température). Le miroir parabolique doré $\mathrm{M}_{3}$ est utilisé pour collecter le rayonnement infrarouge émis et le concentrer sur les détecteurs. Un système d'amplification spécifique est associé à chaque détecteur afin d'optimiser le rapport signal sur bruit. Le signal analogique délivré par le détecteur est d'abord amplifié, grâce à un dispositif en pont de résistance ou à un convertisseur courant/tension, selon le type de détecteur utilisé (photoconducteur ou photovoltaïque). La ligne de base (signal constant avant l'impulsion) est alors soustraite en utilisant un amplificateur différentiel. Le signal est finalement filtré par un filtre passe-bas ayant une fréquence de coupure de $30 \mathrm{kHz}$, avant d'être numérisé par le convertisseur analogiquenumérique d'une carte d'acquisition de données. Tous les paramètres de l'acquisition de données (gains d'amplification, fréquence, nombre de points d'acquisition, valeur de pré-trigger, etc.) sont choisis par l'utilisateur à partir d'une interface homme-machine Labview. Le début de l'acquisition des données est synchronisé avec l'impulsion laser en utilisant un signal de déclenchement généré par la carte d'acquisition. Les signaux provenant des détecteurs avant le signal déclenchement (correspondant à la ligne de base du thermogramme) sont stockés en permanence dans une mémoire tampon circulaire. Lorsque le signal de déclenchement est détecté, les nouvelles données sont stockées dans une mémoire «postdéclenchement $»$.

\subsection{Mesure en face avant}

Un nouveau système optique de détection (Fig. 1) a été installé sur le banc existant afin de mesurer l'évolution de la température de la face avant, à l'emplacement de la perturbation thermique [15]. Le faisceau laser, qui a un diamètre initial d'environ $12 \mathrm{~mm}$, est focalisé par la lentille $\mathrm{L}_{1}$ en $\mathrm{BK} 7$ quelques millimètres avant le miroir plan $\mathrm{M}_{1}$ puis passe par un trou (diamètre de $5 \mathrm{~mm}$ ) situé au centre du miroir $\mathrm{M}_{2}$. Il traverse le hublot $\mathrm{H}_{1}$ et diverge dans le four pour irradier l'ensemble de la face avant de l'échantillon. Le rayonnement infrarouge émis par l'échantillon, consécutivement à cette impulsion de flux, traverse le hublot $\mathrm{H}_{1}$ et est dirigé par le miroir plan $\mathrm{M}_{2}$ vers le détecteur. Il est ensuite focalisé par la lentille 


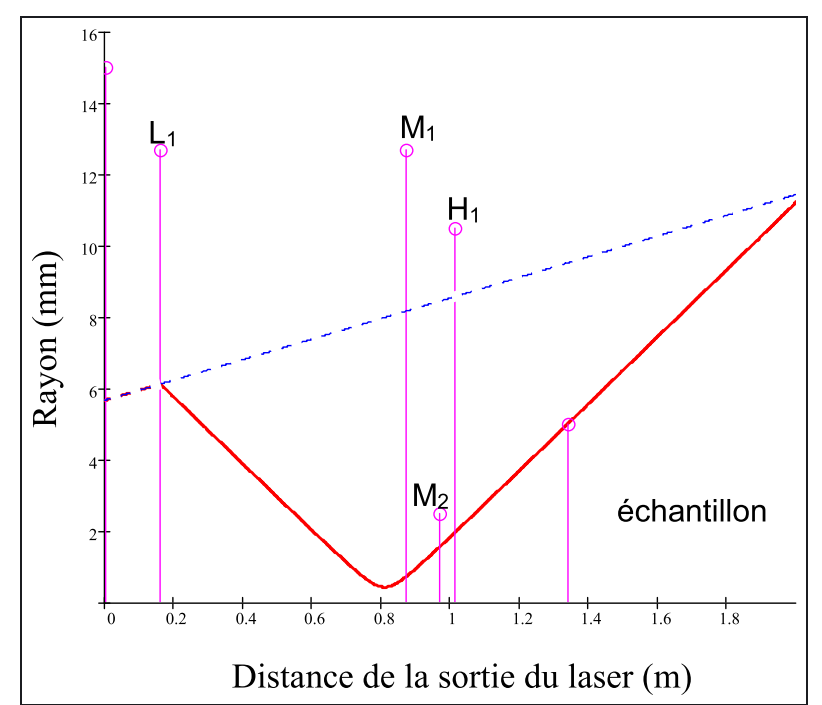

Fig. 2. - Trajet et dimension du faisceau laser.

$\mathrm{L}_{2}$ en $\mathrm{ZnSe}$ sur l'élément sensible du détecteur (diamètre de $1 \mathrm{~mm}$ ). Ainsi, la zone «vue» par le détecteur est l'image de l'élément sensible sur l'échantillon.

L'alignement des éléments optiques (lentilles, miroirs, etc.) est réalisé avec un laser $\mathrm{HeNe}$ concentrique avec le laser $\mathrm{Nd}$ :phosphate. La figure 2 présente le trajet optique du laser compte tenu du choix des optiques et de leur positionnement. L'axe de propagation du faisceau est représenté par l'axe des abscisses. L'axe des ordonnées représente les dimensions (rayon) du faisceau et des optiques. Le tracé pointillé représente le «bord » du faisceau, pour un diamètre de faisceau contenant $86,5 \%$ de la puissance totale dans le cas d'un faisceau gaussien, tel qu'il se propage librement (sans composant optique). Le tracé en trait plein représente le «bord» du faisceau sur le même trajet mais en présence des composants optiques : de gauche à droite, $\mathrm{L}_{1}$, lentille convergente, $\mathrm{M}_{1}$, miroir de renvoi, $\mathrm{M}_{2}$, miroir de renvoi troué, $\mathrm{H}_{1}$, hublot et échantillon.

\section{Validation du dispositif de mesure en face avant}

\subsection{Validation théorique du système optique par simulation optique}

La configuration optique a été validée par des simulations optiques (méthode par tracé de rayons). Elles ont montré qu'en inclinant le hublot $\mathrm{H}_{1}$ de quelques degrés $\left(4^{\circ}\right)$ par rapport à l'horizontal, les réflexions parasites du faisceau laser sur ce hublot (éventuellement présentes malgré le traitement anti-reflet de celui-ci) ne passent pas par la lentille $L_{2}$ et ne sont donc pas focalisées sur le détecteur. Ceci réduit les risques d'avoir un pic parasite sur le thermogramme, voire d'endommager le détecteur. Par ailleurs, le rayonnement émis par l'échantillon est très majoritairement focalisé sur l'élément sensible du détecteur infrarouge et seule une petite partie passe à travers le trou du miroir $\mathrm{M}_{2}$.

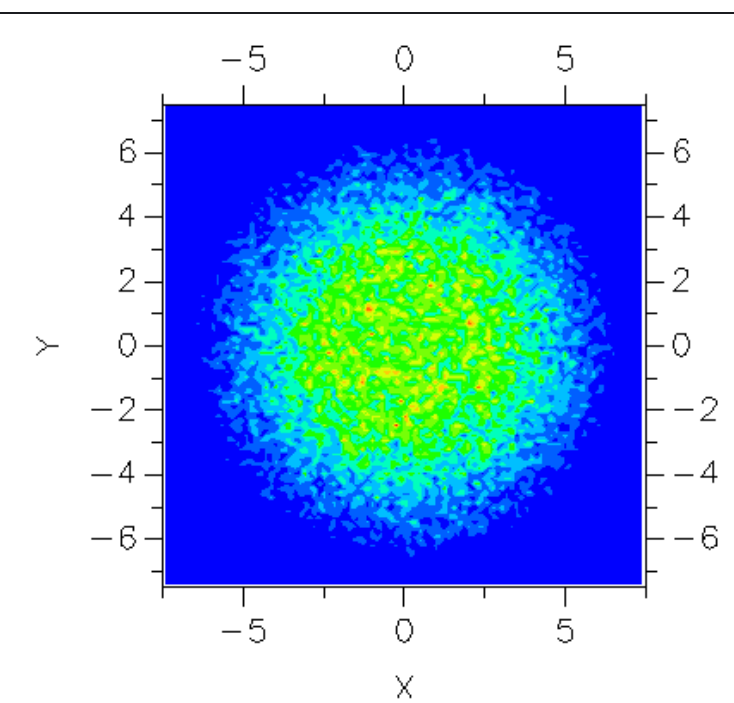

(a) éclairement du laser sur l'échantillon.

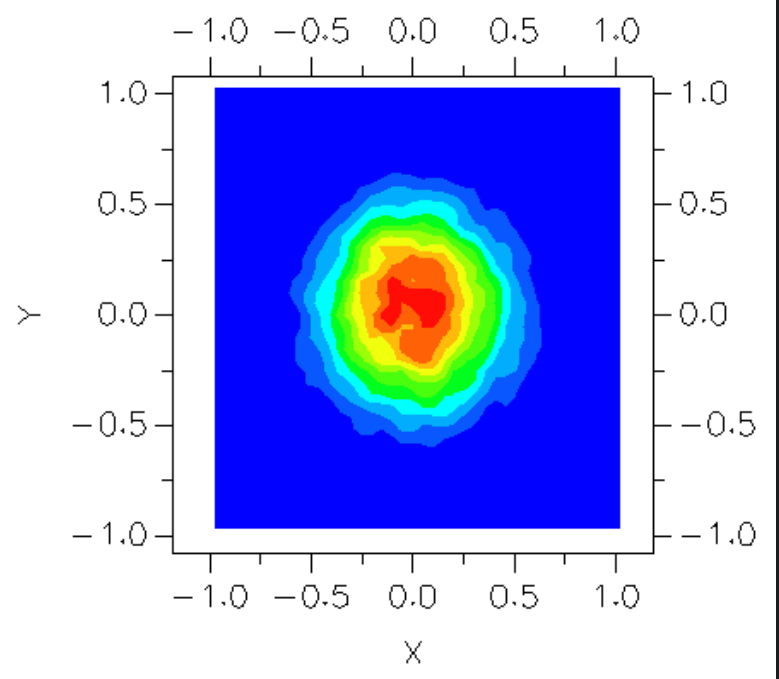

(b) rayonnement émis par l'échantillon sur le détecteur.

Fig. 3. - Cartographies d'éclairement dans le plan de l'échantillon (a) et du détecteur (b) ; $x$ et $y$ sont exprimés en millimètres.

Ces simulations ont également permis de faire des cartographies d'éclairements dans le plan de l'échantillon et du détecteur, en considérant que l'échantillon se comporte comme un corps noir à une température d'environ $1000 \mathrm{~K}$ sur la bande spectrale de $2 \mu \mathrm{m}$ à $12 \mu \mathrm{m}$. Elles montrent que le laser «éclaire» toute la surface de l'échantillon (Fig. 3a) et que l'image de l'échantillon par la lentille $\mathrm{L}_{2}$ a un diamètre de $1 \mathrm{~mm}$ dans le plan du détecteur équivalent à celui de ce dernier (Fig. 3b).

\subsection{Validation expérimentale par la mesure de diffusivité de matériaux homogènes}

La validation de la méthode «flash» en face avant a été réalisée en mesurant la diffusivité thermique de deux matériaux homogènes bien connus : le fer Armco et le graphite Poco AXM-5Q1. Les figures 4 et 5 présentent des thermogrammes expérimentaux (en échelles 


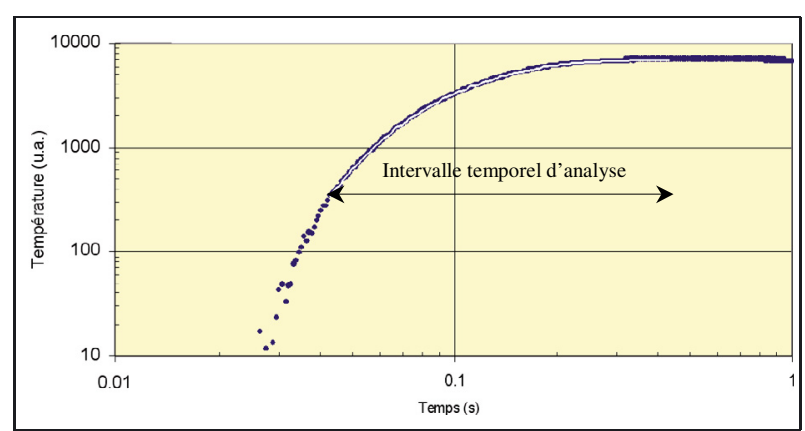

Fig. 4. - Thermogramme obtenu à $400{ }^{\circ} \mathrm{C}$ en face arrière d'un échantillon de fer Armco.

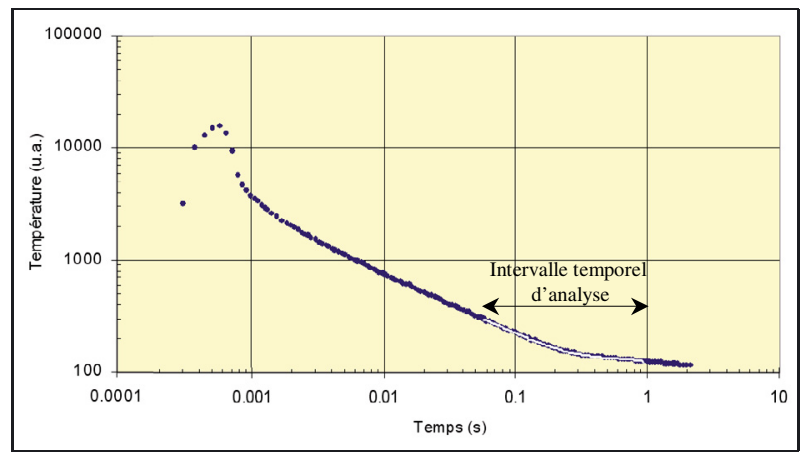

Fig. 5. - Thermogramme obtenu à $400{ }^{\circ} \mathrm{C}$ en face avant d'un échantillon de fer Armco.

logarithmiques), obtenus à $400{ }^{\circ} \mathrm{C}$ respectivement en faces avant et arrière d'un échantillon de fer Armco. L'intervalle temporel d'analyse, sur lequel l'estimation des paramètres a été effectuée conformément aux travaux de Krapez $[11,12]$, est représenté sur les figures $4,5,7$ et 8 par une double flèche.

La figure 4 montre l'allure «classique » d'un thermogramme « face arrière », où le signal maximum est atteint environ $0,5 \mathrm{~s}$ après l'apport d'énergie et est suivi par une très faible diminution en raison des pertes thermiques. Le thermogramme présenté sur la figure 5 montre que le signal maximum, atteint $0,6 \mathrm{~ms}$ après l'apport d'énergie sur la face avant, est suivi d'une décroissance de pente en $t^{-1 / 2}$, caractéristique du comportement thermique d'un milieu semi-infini. Lorsque la chaleur atteint la face arrière de l'échantillon, un « coude » apparaît à 0,3 s puis est suivi par une évolution quasi-adiabatique. En fait, la température de la face avant continue de diminuer en raison des pertes thermiques sur les deux faces. C'est approximativement la localisation temporelle de ce «coude», qui permet l'identification de la diffusivité thermique d'un matériau homogène. La partie initiale des thermogrammes est généralement affectée par des effets parasites (opacité imparfaite du matériau, non-uniformité de chauffage, parasite électromagnétique... ). D'autres effets viennent quant à eux faire diverger les thermogrammes au temps longs (pertes latérales, dérive...). En conséquence, l'intervalle temporel d'analyse encadre le «coude» sur environ une décade de temps à une décade et demie, tout en évitant les portions du thermogramme correspondant aux temps courts et aux temps longs.
Les tableaux 1 et 2 présentent les résultats de mesure de la diffusivité thermique obtenus sur des éprouvettes de fer Armco et de graphite Poco AXM-5Q1 respectivement. Ces résultats sont la moyenne de trois mesures successives effectuées dans les mêmes conditions expérimentales. La colonne identifiée (1) donne des valeurs provenant d'expressions polynomiales, déterminées par le LNE [7] à partir des résultats rapportés par plusieurs auteurs [16-21].

Les mesures effectuées sur la face arrière ont été analysées en utilisant les deux méthodes d'identification décrites précédemment : la méthode des moments temporels partiels et la méthode de minimisation proposée par Krapez. L'objectif de cette approche est de comparer cette dernière méthode avec une méthode éprouvée dans le cas de matériaux connus. Ces deux méthodes sont référencées respectivement «Moment » et «Minim. » dans les tableaux 1 et 2. La colonne identifiée (2) donne l'écart relatif entre les résultats obtenus par les deux méthodes. Dans le cas de mesures effectuées sur la face avant, seule la méthode de minimisation est utilisée car la méthode des moments temporels partiels ne peut pas s'appliquer à cette configuration de mesure. La colonne identifiée (3) donne l'écart relatif entre les résultats obtenus avec la méthode de minimisation sur la face avant et sur la face arrière.

Les variations relatives entre toutes nos mesures et les valeurs obtenues à partir des expressions polynomiales sont inférieures à $2 \%$ pour le fer Armco et inférieures à $3 \%$ pour le graphite Poco AXM-5Q1 (sauf à $23{ }^{\circ} \mathrm{C}$, où cette variation relative est de $6,5 \%$ ). L'écart relatif (2) entre les résultats obtenus par les deux méthodes d'identification est inférieur à $2 \%$ (sauf pour le graphite à $23{ }^{\circ} \mathrm{C}$ ) sur toute la plage des températures entre $23{ }^{\circ} \mathrm{C}$ et $1400^{\circ} \mathrm{C}$ pour les deux matériaux homogènes. On peut considérer que ces deux méthodes conduisent à des résultats identiques, l'incertitude relative élargie $(k=2)$ sur les mesures de diffusivité thermique du fer Armco et du graphite Poco par la méthode des moments temporels partiels étant estimée entre $\pm 3 \%$ et $\pm 5,5 \%$ sur la gamme des températures entre $23^{\circ} \mathrm{C}$ et $1400{ }^{\circ} \mathrm{C}$ [22,23]. Les résultats de mesure montrent aussi une bonne cohérence avec un écart relatif (3) de moins de 3,5\% entre les valeurs obtenues par les méthodes en face avant et en face arrière. La répétabilité sur trois mesures est meilleure que $1,5 \%$ jusqu'à $1400{ }^{\circ} \mathrm{C}$ pour les mesures en face arrière. Elle est inférieure à $2 \%$ en dessous de $800{ }^{\circ} \mathrm{C}$, et meilleure que $3 \%$ pour les températures entre $800^{\circ} \mathrm{C}$ et $1400{ }^{\circ} \mathrm{C}$ pour les mesures en face avant.

\section{Caractérisation thermique de dépôts céramiques}

\subsection{Mesure de la diffusivité thermique}

Après avoir validé la méthode «flash » en face avant dans le cas de matériaux homogènes, les deux méthodes «face avant» et «face arrière » ont été appliquées à des matériaux multicouches, constitués d'une couche 
Tableau 1

Comparaison des mesures en face avant et en face arrière pour le Fer Armco.

\begin{tabular}{|c|c|c|c|c|c|c|}
\hline \multicolumn{3}{|c|}{ FER ARMCO } & \multicolumn{3}{c|}{ Epaisseur $=2,802 \mathrm{~mm}$ à $23^{\circ} \mathrm{C}$} \\
\hline \multirow{3}{*}{ Temp. $\left({ }^{\circ} \mathrm{C}\right)$} & \multicolumn{5}{c|}{ Diffusivité thermique $\left(10^{-6} \mathrm{~m}^{2} \cdot \mathrm{s}^{-1}\right)$} \\
\cline { 2 - 7 } & \multirow{2}{*}{ Réf. 16-21 (1) } & \multicolumn{3}{|c|}{ Face arrière } & \multicolumn{2}{c|}{ Face avant } \\
\cline { 3 - 7 } & & Moment & Minim. & Écart (2) $(\%)$ & Minim. & Écart (3) (\%) \\
\hline 23 & 20,25 & 20,47 & 20,35 & 0,6 & 20,24 & 0,5 \\
\hline 200 & 14,40 & 14,54 & 14,56 & $-0,1$ & 14,47 & 0,6 \\
\hline 400 & 9,93 & 10,02 & 10,04 & $-0,1$ & 10,14 & $-1,0$ \\
\hline 600 & 6,57 & 6,56 & 6,51 & 0,7 & 6,47 & 0,7 \\
\hline 800 & 4,48 & 4,47 & 4,41 & 1,4 & 4,45 & $-1,0$ \\
\hline
\end{tabular}

Tableau 2

Comparaison des mesures en face avant et en face arrière pour le graphite Poco.

\begin{tabular}{|c|c|c|c|c|c|c|}
\hline \multicolumn{5}{|c|}{ GRAPHITE POCO AXM-5Q1 } & \multicolumn{3}{c|}{ Epaisseur $=2,991 \mathrm{~mm}$ à $23{ }^{\circ} \mathrm{C}$} \\
\hline \multirow{3}{*}{ Temp. $\left({ }^{\circ} \mathrm{C}\right)$} & \multicolumn{5}{|c|}{ Diffusivité thermique $\left(10^{-6} \mathrm{~m}^{2} \cdot \mathrm{s}^{-1}\right)$} \\
\cline { 2 - 7 } & \multirow{2}{*}{ Réf. 16-21 1 } & \multicolumn{3}{|c|}{ Face arrière } & \multicolumn{2}{c|}{ Face avant } \\
\cline { 2 - 7 } & & Moment & Minim. & Écart (2) $(\%)$ & Minim. & Écart (3) (\%) \\
\hline 23 & 81,22 & 78,76 & 75,91 & 3,7 & 76,22 & $-0,4$ \\
\hline 200 & 45,17 & 44,62 & 44,04 & 1,3 & 45,54 & $-3,3$ \\
\hline 400 & 29,42 & 28,62 & 28,61 & 0,0 & 29,25 & $-2,2$ \\
\hline 600 & 22,48 & 21,97 & 21,89 & 0,3 & 22,51 & $-2,8$ \\
\hline 800 & 18,08 & 17,87 & 17,87 & 0,0 & 18,05 & $-1,0$ \\
\hline 1000 & 15,19 & 15,06 & 14,82 & 1,6 & 15,28 & $-3,1$ \\
\hline 1200 & 13,55 & 13,35 & 13,29 & 0,5 & 13,30 & $-0,1$ \\
\hline 1400 & 12,22 & 12,24 & 12,04 & 1,7 & 12,34 & $-2,5$ \\
\hline
\end{tabular}

d'oxyde de chrome déposée par projection plasma sur un substrat en alliage de fer.

Les dépôts d'oxyde de chrome sont très résistants à l'usure [24], à l'abrasion en milieu agressif, et à la corrosion à haute température [25]. Ces types de revêtements céramiques épais sont en particulier utilisés pour les composants de moteurs diesels automobiles ou marins, tels que les têtes de piston, les soupapes d'échappement, et les chemises de cylindre [26]. Par exemple, le constructeur d'automobiles Renault a appliqué des dépôts d'oxyde de chrome obtenus par projection plasma à des segments de piston [27].

Des mesures ont été effectuées entre $23{ }^{\circ} \mathrm{C}$ et $800{ }^{\circ} \mathrm{C}$ sur les deux systèmes «revêtement/substrat» suivants :

- A : 0,229 mm de revêtement de $\mathrm{Cr}_{2} \mathrm{O}_{3}$ sur un substrat d'alliage de fer d'épaisseur 0,997 mm ;

- B : 0,405 mm de revêtement de $\mathrm{Cr}_{2} \mathrm{O}_{3}$ sur un substrat d'alliage de fer d'épaisseur $6,000 \mathrm{~mm}$.

Avant d'effectuer ces mesures, un premier échantillon avait été chauffé progressivement par pas de $100{ }^{\circ} \mathrm{C}$ afin de déterminer la température d'utilisation maximale du revêtement. Des fissures sont apparues sur le dépôt à partir de $900{ }^{\circ} \mathrm{C}$ (Fig. 6), résultant de la relaxation des contraintes résiduelles au cours du cyclage thermique [28]. Il a donc été décidé de ne pas effectuer des

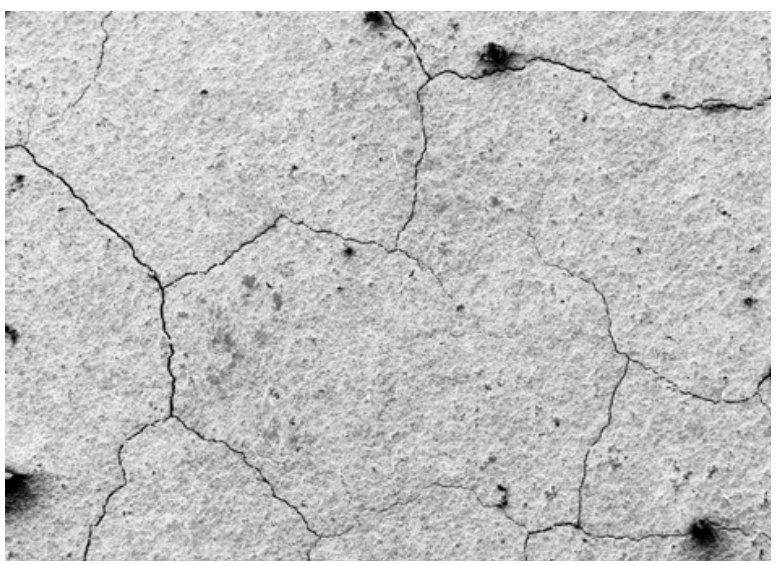

Fig. 6. - Micrographie MEB du revêtement d'oxyde de chrome après chauffage à $900{ }^{\circ} \mathrm{C}$ (grossissement $\left.\times 25\right)$.

mesures au-delà de $800{ }^{\circ} \mathrm{C}$ sur les échantillons étudiés, afin d'éviter d'endommager le revêtement.

Les propriétés thermiques du substrat doivent être connues afin de permettre la caractérisation de la diffusivité thermique du revêtement. Les dilatation thermique, densité, capacité thermique massique, et diffusivité thermique du substrat ont donc été préalablement mesurées de $23{ }^{\circ} \mathrm{C}$ à $800{ }^{\circ} \mathrm{C}$ par le LNE sur les substrats nus, respectivement, par dilatométrie à poussoir, méthode par 
Tableau 3

Propriétés thermiques du substrat en alliage de fer.

\begin{tabular}{|c|c|c|c|c|}
\hline $\begin{array}{c}\text { Temp. } \\
\left({ }^{\circ} \mathrm{C}\right)\end{array}$ & $\begin{array}{c}a \\
\left(10^{-6} \mathrm{~m}^{2} \cdot \mathrm{s}^{-1}\right)\end{array}$ & $\begin{array}{c}\alpha \\
\left(10^{-6} \mathrm{~K}^{-1}\right)\end{array}$ & $\begin{array}{c}\rho \\
\left(\mathrm{kg} \cdot \mathrm{m}^{-3}\right)\end{array}$ & $\begin{array}{c}c_{p} \\
\left(\mathrm{~J} \cdot \mathrm{kg}^{-1} \cdot \mathrm{K}^{-1}\right)\end{array}$ \\
\hline 23 & 16,53 & - & 7814 & 463 \\
200 & 13,22 & 14,1 & 7756 & 517 \\
400 & 9,72 & 14,5 & 7687 & 585 \\
600 & 6,46 & 15,0 & 7614 & 696 \\
800 & 4,47 & 15,4 & 7540 & 798 \\
\hline
\end{tabular}

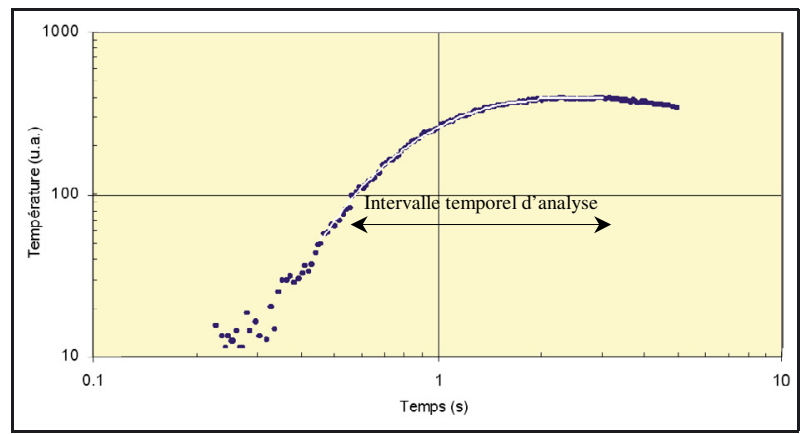

Fig. 7. - Thermogramme obtenu à $600{ }^{\circ} \mathrm{C}$ sur la face arrière d'un matériau multicouche $\mathrm{Cr}_{2} \mathrm{O}_{3} / \mathrm{Fe}$.

immersion, calorimétrie différentielle à balayage, et méthode «flash laser» en face arrière (Tab. 3).

Les figures 7 et 8 donnent des exemples de thermogrammes expérimentaux (échelles logarithmiques) obtenus à $600{ }^{\circ} \mathrm{C}$, respectivement sur les faces avant et arrière du système $\mathrm{B}$. Le thermogramme obtenu en face arrière, situé sur la figure 7, a une apparence assez semblable à celui d'un matériau homogène (Fig. 4), sans aucun signe distinctif visible en relation avec l'hétérogénéité des propriétés thermiques d'un multicouche.

En revanche, les thermogrammes obtenus en face avant diffèrent nettement entre un matériau homogène et un multicouche. La figure 8 montre, comme dans le cas des matériaux homogènes (Fig. 5), que la température suit d'abord une diminution de pente en $t^{-1 / 2}$, comme si le revêtement était seul. Mais après un premier coude, situé vers $0,1 \mathrm{~s}$ correspondant au temps où la chaleur atteint le substrat, la diminution s'accélère jusqu'à un second coude situé autour de $1 \mathrm{~s}$. Après ce coude, qui représente l'instant où la chaleur atteint la face arrière du matériau multicouche, la température approche d'un palier adiabatique correspondant au cas d'un matériau multicouche sans perte. Comme précédemment, le matériau multicouche est soumis à des pertes thermiques, la température de la face avant continue en fait de diminuer. La diffusivité thermique du revêtement est identifiée par la localisation temporelle de cette zone de transition.

Le tableau 4 présente une comparaison des résultats de mesure de la diffusivité thermique obtenus en face avant et en face arrière pour les deux systèmes multicouches $\mathrm{A}$ et $\mathrm{B}$ de $\mathrm{Cr}_{2} \mathrm{O}_{3} / \mathrm{Fe}$. Les valeurs obtenues sont en bon accord avec celles données dans les publications [24,29]. L'écart relatif entre les résultats obtenus par

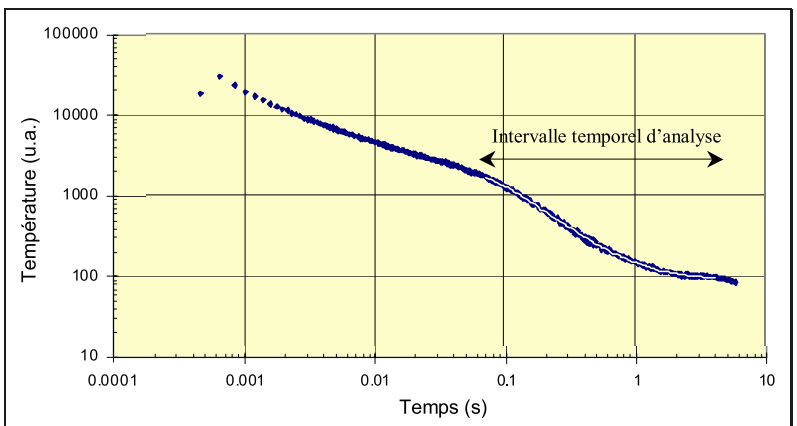

Fig. 8. - Thermogramme obtenu à $600{ }^{\circ} \mathrm{C}$ sur la face avant d'un matériau multicouche $\mathrm{Cr}_{2} \mathrm{O}_{3} / \mathrm{Fe}$.

les deux méthodes est au maximum de $5 \%$ sur la plage des températures entre $23{ }^{\circ} \mathrm{C}$ et $800{ }^{\circ} \mathrm{C}$, pour les deux multicouches. Toutefois, cet écart est plus important pour le matériau $\mathrm{B}\left(\mathrm{Cr}_{2} \mathrm{O}_{3} / \mathrm{Fe}\right.$ de $\left.0,405 \mathrm{~mm} / 6,000 \mathrm{~mm}\right)$ que pour le matériau A $\left(\mathrm{Cr}_{2} \mathrm{O}_{3} / \mathrm{Fe}\right.$ de $\left.0,229 \mathrm{~mm} / 0,997 \mathrm{~mm}\right)$. La répétabilité sur trois mesures consécutives est inférieure à $2 \%$ pour les mesures effectuées sur la face avant des deux multicouches, et sur la face arrière du matériau A. Elle croît jusqu'à $7 \%$ pour les mesures effectuées sur la face arrière des matériaux multicouches B. Ces observations peuvent s'expliquer par un amortissement plus élevé du signal thermique pour le multicouche épais que pour le plus mince. Cela conduit inévitablement à un plus mauvais rapport signal sur bruit des mesures réalisées en face arrière pour le matériau $B$ que pour le matériau $A$. Ces résultats corroborent les conclusions de l'étude de sensibilité de la méthode $[11,12]$.

Les résultats présentés dans le tableau 4, mettent en évidence une augmentation inhabituelle de la diffusivité thermique de l'oxyde de chrome avec les cycles thermiques. Les valeurs acquises à $23{ }^{\circ} \mathrm{C}$ après cyclage sont plus élevées que celles obtenues initialement (en hausse de près de $30 \%$ pour le multicouche $A$ ). Ce phénomène est probablement dû à une modification de la céramique par frittage vers $500{ }^{\circ} \mathrm{C}$ et à sa densification au cours du cycle de chauffage. Un comportement similaire a été observé par Zhu [30] sur un dépôt de $\mathrm{ZrO}_{2}-8 \% \mathrm{Y}_{2} \mathrm{O}_{3}$ obtenu par projection plasma. En outre, les valeurs de la diffusivité thermique obtenue avec le matériau composite A sont un peu supérieures à celles déterminées avec le matériau composite $\mathrm{B}$.

Après avoir effectué les mesures de diffusivité thermique, nous avons étudié la microstructure des deux revêtements par microscopie optique afin d'expliquer ce comportement. Un système d'imagerie complet, composé d'un microscope optique à fort grossissement couplé à une caméra $\mathrm{CCD}$, a été utilisé. Ce système a été étalonné en longueur afin de mesurer l'épaisseur des revêtements avec une incertitude de quelques micromètres. Les images obtenues, présentées sur la figure 9, montrent clairement que l'aspect du dépôt varie en fonction de son épaisseur et que le revêtement le plus épais contient plus de pores et de fissures que le plus mince (les substrats apparaissant en blanc sur les images au microscope). 
Tableau 4

Comparaison des mesures en face avant et en face arrière sur un matériau multicouche $\mathrm{Cr}_{2} \mathrm{O}_{3} / \mathrm{Fe}$.

\begin{tabular}{|c|c|c|c|c|c|c|}
\hline \multicolumn{7}{|c|}{ REVETEMENT $\mathrm{Cr}_{2} \mathrm{O}_{3}$} \\
\hline \multirow{3}{*}{ Temp. $\left({ }^{\circ} \mathrm{C}\right)$} & \multicolumn{6}{|c|}{ Diffusivité thermique $\left(10^{-6} \mathrm{~m}^{2} \cdot \mathrm{s}^{-1}\right)$} \\
\hline & \multicolumn{3}{|c|}{ Multicouche A } & \multicolumn{3}{|c|}{ Multicouche B } \\
\hline & Face arrière & Face avant & Écart (\%) & Face arrière & Face avant & Écart (\%) \\
\hline 23 & 1,283 & 1,292 & $-0,7$ & 1,325 & 1,263 & 5,0 \\
\hline 200 & 1,084 & 1,100 & $-1,5$ & 0,992 & 0,954 & 3,9 \\
\hline 400 & 0,877 & 0,895 & $-2,0$ & 0,756 & 0,779 & $-3,1$ \\
\hline 600 & 0,856 & 0,877 & $-2,4$ & 0,773 & 0,783 & $-1,1$ \\
\hline 800 & 0,840 & 0,860 & $-2,4$ & 0,831 & 0,809 & 2,7 \\
\hline 23 & 1,635 & 1,672 & $-2,3$ & 1,373 & 1,319 & 4,0 \\
\hline
\end{tabular}

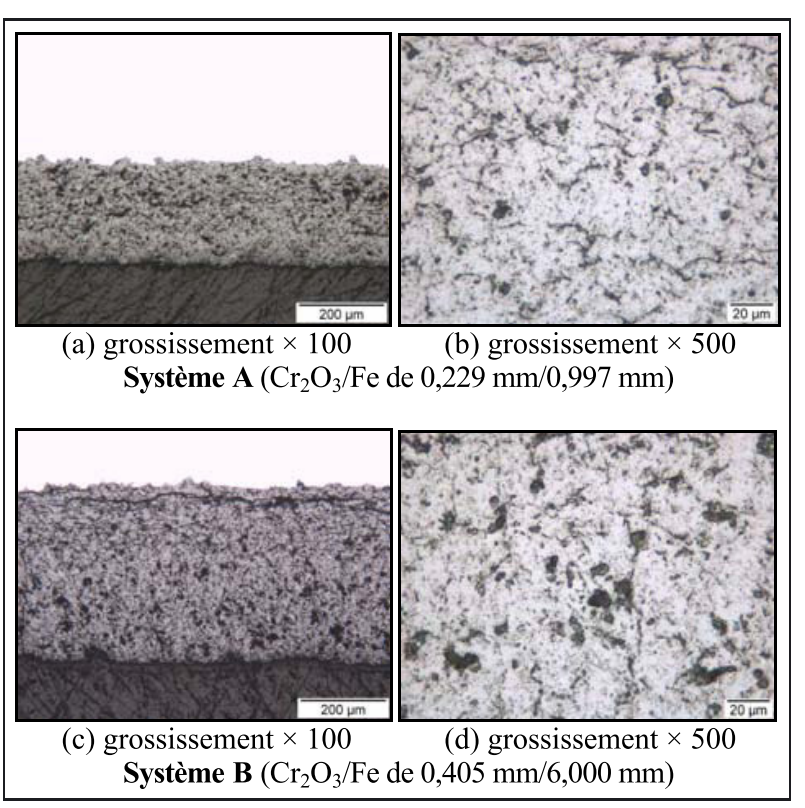

Fig. 9. - Microstructures des revêtements d'oxyde de chrome des systèmes A et B.

Les revêtements appliqués par pulvérisation plasma, ont une microstructure lamellaire et présentent souvent plusieurs types de défauts (non-homogénéité de structure, porosité ouverte, fissures, etc.) liés à la technique de dépôt $[31,32]$. Ces fissures, résultant de la relaxation des contraintes résiduelles au cours des cycles thermiques, sont d'autant plus nombreuses que le revêtement est épais [28]. La légère différence de microstructure des revêtements pourrait expliquer la différence entre les valeurs de la diffusivité thermique obtenues pour les deux revêtements.

\subsection{Détermination simultanée de la diffusivité thermique et de la conductivité thermique}

La procédure d'estimation de paramètre développée par Krapez [12] a également été appliquée à la détermination simultanée de la diffusivité thermique et de la conductivité thermique du revêtement d'oxyde de chrome. L'intervalle temporel d'analyse a été modifié (cf. Fig. 10 comparativement à la Fig. 8) pour que

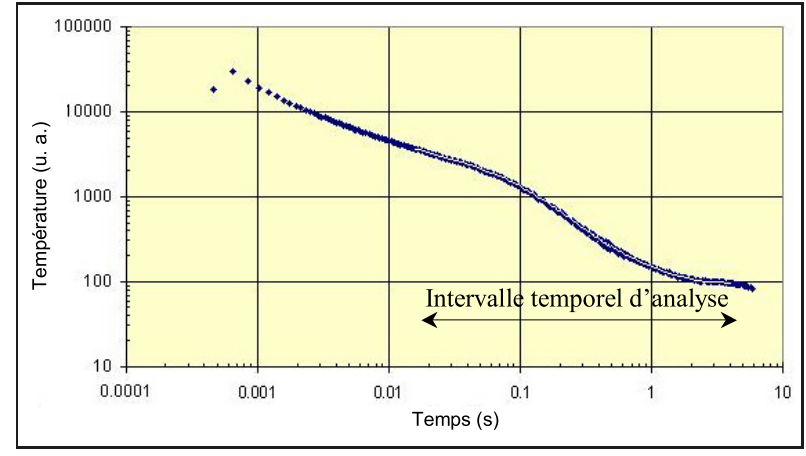

Fig. 10. - Thermogramme obtenu à $600{ }^{\circ} \mathrm{C}$ sur la face avant d'un matériau multicouche $\mathrm{Cr}_{2} \mathrm{O}_{3} / \mathrm{Fe}$.

l'incertitude des estimateurs des paramètres recherchés (diffusivité thermique et l'effusivité thermique) soit minimisée, tout en évitant une corrélation forte entre eux.

La diffusivité thermique est estimée à partir de la localisation temporelle de la zone de transition et l'effusivité thermique à partir de son amplitude verticale. Le bicouche étudié ici n'est qu'un cas particulier. Il est important de remarquer que dans le cas où le revêtement n'est plus un isolant et que le substrat n'est plus un conducteur (ou plutôt un matériau effusif) les courbes des figures 8 et 10 n'ont plus la même allure.

Le tableau 5 présente les valeurs de la diffusivité thermique et de l'effusivité thermique mesurées simultanément sur le revêtement de $\mathrm{Cr}_{2} \mathrm{O}_{3}$ du matériau multicouche $\mathrm{B}$ entre $23{ }^{\circ} \mathrm{C}$ et $800^{\circ} \mathrm{C}$. La quatrième colonne donne les résultats pour la conductivité thermique calculée à partir de ces deux propriétés grâce à la relation (1).

La conductivité thermique du revêtement de $\mathrm{Cr}_{2} \mathrm{O}_{3}$ déterminée à $23{ }^{\circ} \mathrm{C}$ est approximativement égale au tiers de la valeur $\left(\sim 16 \mathrm{~W} \cdot \mathrm{m}^{-1} \cdot \mathrm{K}^{-1}\right)$ mesurée par Bruce [33] et Marinelli [34] sur des échantillons massifs. Là encore, ceci peut être expliqué par la grande porosité et la microstructure spécifique de ce type de revêtements.

Zhu [30] a obtenu des résultats comparables avec un oxyde de zircone stabilisé à l'yttrium. Il a observé qu'un dépôt, d'épaisseur $180 \mu \mathrm{m}$, avait des valeurs de conductivité thermique significativement plus faibles (plus de 
Tableau 5

Diffusivité thermique et conductivité thermique du revêtement de $\mathrm{Cr}_{2} \mathrm{O}_{3}$ (multicouche $\mathrm{B}$ ).

\begin{tabular}{|c|c|c|c|}
\hline $\begin{array}{c}\text { Temp. } \\
\left({ }^{\circ} \mathrm{C}\right)\end{array}$ & $\begin{array}{c}\text { Diffusivité } \\
\text { thermique } \\
\left(10^{-6} \mathrm{~m}^{2} \cdot \mathrm{s}^{-1}\right)\end{array}$ & $\begin{array}{c}\text { Effusivité } \\
\text { thermique } \\
\left(\mathrm{J} \cdot \mathrm{m}^{-2} \cdot \mathrm{K}^{-1} \cdot \mathrm{s}^{-0,5}\right)\end{array}$ & $\begin{array}{c}\text { Conductivité } \\
\text { thermique } \\
\left(\mathrm{W} \cdot \mathrm{m}^{-1} \cdot \mathrm{K}^{-1}\right)\end{array}$ \\
\hline 23 & 1,263 & 4570 & 5,14 \\
200 & 0,954 & 3830 & 3,74 \\
400 & 0,779 & 3600 & 3,18 \\
600 & 0,783 & 3390 & 3,00 \\
800 & 0,809 & 3030 & 2,73 \\
\hline
\end{tabular}

deux fois plus faibles) que celles d'échantillons massifs de même nature.

\subsection{Comparaison de cette méthode de mesure de conductivité thermique avec une approche indirecte}

Cette méthode de mesure a été validée en comparant les valeurs de conductivité thermique obtenues (présentées dans le tableau 5) avec celles déterminées au moyen d'une approche indirecte. Cette méthode consiste à évaluer la conductivité thermique du revêtement à partir de sa diffusivité thermique $a$, sa masse volumique $\rho$ et sa capacité thermique massique $c_{p}$ en utilisant la relation (2).

$$
\lambda=a \cdot \rho \cdot c_{p}
$$

Après avoir mesuré la diffusivité thermique et l'effusivité thermique du revêtement de $23^{\circ} \mathrm{C}$ à $800^{\circ} \mathrm{C}$, le substrat en alliage de fer a été dissous dans de l'acide chlorhydrique $6 \mathrm{~N}$ afin de conserver uniquement la couche de $\mathrm{Cr}_{2} \mathrm{O}_{3}$. La masse volumique du revêtement a été déterminée à $23{ }^{\circ} \mathrm{C}$ selon la méthode par immersion. Elle a été calculée par la relation (3).

$$
\rho_{23{ }^{\circ} \mathrm{C}}=\frac{m_{1} \cdot \rho_{\ell}}{\left(m_{1}-m_{2}\right)},
$$

où $m_{1}$ est la masse apparente de l'échantillon dans l'air, $m_{2}$ est la masse apparente de l'échantillon dans le liquide d'immersion (eau distillée à $23{ }^{\circ} \mathrm{C} \pm 0,1^{\circ} \mathrm{C}$ ) et $\rho_{\ell}$ est la masse volumique du liquide d'immersion à $23^{\circ} \mathrm{C}$. La masse volumique $\rho_{T}$, pour les températures $T$ supérieures à $23^{\circ} \mathrm{C}$, a été calculée avec la formule (4).

$$
\rho_{T}=\frac{\rho_{23}{ }^{\circ} \mathrm{C}}{\left.\left(1+\alpha_{L}\right]_{T_{0}}^{T} \cdot\left(T-T_{0}\right)\right)^{3}}
$$

Les coefficients moyens de dilatation linéique $\left.\alpha_{L}\right]_{T_{0}}^{T}$ entre $T_{0}\left(=23{ }^{\circ} \mathrm{C}\right)$ et $T$, qui ont servi à calculer la masse volumique en fonction de la température, proviennent de [24]. La masse volumique mesurée à $23{ }^{\circ} \mathrm{C}$ (Tab. 6) est proche de la valeur (4 $900 \mathrm{~kg} \cdot \mathrm{m}^{-3}$ ) donnée par Mann pour un revêtement de $\mathrm{Cr}_{2} \mathrm{O}_{3}$ analogue [35]. La capacité thermique massique a été mesurée sous atmosphère d'azote entre $23{ }^{\circ} \mathrm{C}$ et $800^{\circ} \mathrm{C}$ en utilisant un calorimètre différentiel à balayage. Ces mesures ont été effectuées sur $275 \mathrm{mg}$ de
Tableau 6

Comparaison des méthodes de détermination de la conductivité thermique du dépôt de $\mathrm{Cr}_{2} \mathrm{O}_{3}$.

\begin{tabular}{|c|c|c|c|c|c|}
\hline $\begin{array}{c}\text { Temp. } \\
\left({ }^{\circ} \mathrm{C}\right)\end{array}$ & $\begin{array}{c}a \\
\left(10^{-6} \mathrm{~m}^{2} \cdot \mathrm{s}^{-1}\right)\end{array}$ & $\begin{array}{c}c_{p} \\
\left(\mathrm{~J} \cdot \mathrm{kg}^{-1} \cdot \mathrm{K}^{-1}\right)\end{array}$ & $\begin{array}{c}\rho \\
\left(\mathrm{kg} \cdot \mathrm{m}^{-3}\right)\end{array}$ & $\begin{array}{c}\lambda \\
\left(\mathrm{W} \cdot \mathrm{m}^{-1} \cdot \mathrm{K}^{-1}\right)\end{array}$ & $\begin{array}{c}\text { Écart } \\
(\%)\end{array}$ \\
\hline 23 & 1,263 & 822 & 4975 & 5,17 & 0,6 \\
200 & 0,954 & 750 & 4959 & 3,55 & $-5,2$ \\
400 & 0,779 & 773 & 4939 & 2,97 & $-6,8$ \\
600 & 0,783 & 760 & 4920 & 2,93 & $-2,4$ \\
800 & 0,809 & 733 & 4907 & 2,91 & 6,4 \\
\hline
\end{tabular}

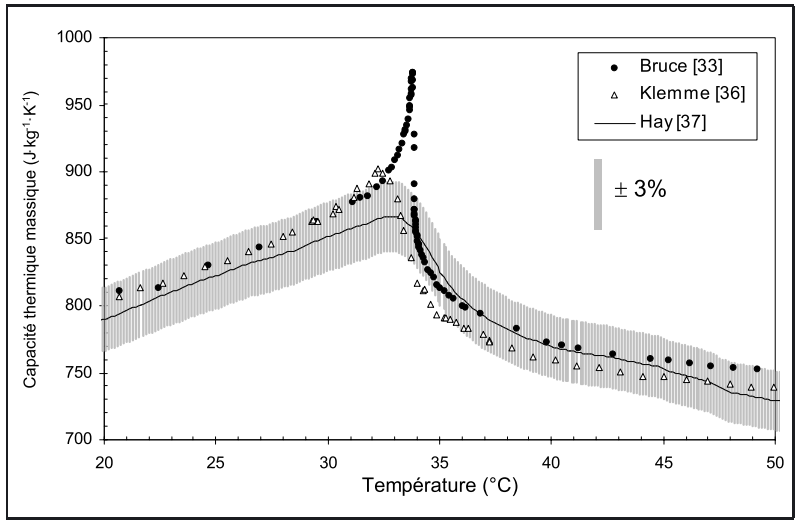

Fig. 11. - Capacité thermique massique du $\mathrm{Cr}_{2} \mathrm{O}_{3}$ au voisinage de la transition de Néel en fonction de la température.

revêtement en appliquant une «méthode étagée » consistant à diviser la plage totale de températures en intervalles de $5 \mathrm{~K}$, et à les parcourir progressivement à une vitesse de chauffage de $5 \mathrm{~K} \cdot \mathrm{min}^{-1}$.

Des mesures complémentaires ont été réalisées entre $20{ }^{\circ} \mathrm{C}$ et $50{ }^{\circ} \mathrm{C}$ par une méthode de balayage continue avec une vitesse de chauffage de $1 \mathrm{~K} \cdot \mathrm{min}^{-1}$, afin de mesurer la capacité thermique massique de la couche de $\mathrm{Cr}_{2} \mathrm{O}_{3}$ au voisinage de la transition de Néel avec une meilleure résolution. Cette transition de l'état paramagnétique à l'état antiferromagnétique du $\mathrm{Cr}_{2} \mathrm{O}_{3}$, qui se produit à environ $33{ }^{\circ} \mathrm{C}$, induit une forte variation de la capacité thermique massique. La figure 11 montre que nos résultats (qui incluent des barres d'incertitude de $\pm 3 \%$ ) sont en bon accord avec ceux obtenus par Bruce [33] et Klemme [36] sur un échantillon massif de $\mathrm{Cr}_{2} \mathrm{O}_{3}$.

Contrairement à la conductivité thermique et à la diffusivité thermique, qui dépendent fortement de la microstructure du matériau étudié (voir commentaires précédents), la capacité thermique massique est principalement influencée par la composition chimique du matériau. Les capacités thermiques massiques d'un revêtement et d'un échantillon massif d'un même matériau ont ainsi une évolution similaire avec la température.

Le tableau 6 présente la conductivité thermique du revêtement de $\mathrm{Cr}_{2} \mathrm{O}_{3}$ en fonction de la température déterminée par méthode indirecte. La dernière colonne donne l'écart relatif entre ces valeurs et celles déterminées à l'aide de la méthode « flash » en face avant (données dans 
la quatrième colonne du Tab. 5). Cet écart relatif est inférieur à $7 \%$ sur la gamme de températures entre $23{ }^{\circ} \mathrm{C}$ et $800^{\circ} \mathrm{C}$. L'incertitude élargie sur les mesures de conductivité thermique de tels revêtements, déterminées selon les deux méthodes, est estimée entre $\pm 6 \%$ et $\pm 10 \%$ selon la température. On peut donc considérer que les deux méthodes conduisent à des résultats identiques.

\section{Conclusion}

Le diffusivimètre de référence du LNE a été modifié, tant sur le plan expérimental (nouveaux systèmes optiques et systèmes de détection) que sur le plan de l'analyse des résultats (adaptation d'une nouvelle méthode d'identification), afin de pouvoir mesurer simultanément la diffusivité thermique et l'effusivité thermique de revêtements à haute température, au moyen de la méthode «flash» en face avant.

La validation de cette méthode a été réalisée en mesurant la diffusivité thermique du fer Armco et du graphite Poco AXM-5Q1 entre $23^{\circ} \mathrm{C}$ et $1400{ }^{\circ} \mathrm{C}$, puis en comparant les résultats obtenus avec ceux déterminés par la méthode «flash » en face arrière. Cette phase de validation a clairement indiqué que les deux méthodes conduisaient à des résultats identiques pour des matériaux homogènes, les valeurs obtenues différant de moins de 3,5\%, avec une incertitude relative élargie inférieure à 5,5\%.

Les deux méthodes ont ensuite été mises en œuvre pour la mesure de la diffusivité thermique de deux revêtements en oxyde de chrome d'épaisseurs différentes, déposés sur un substrat en alliage de fer. Les valeurs obtenues selon les deux méthodes sont en bon accord, avec un écart relatif inférieur à $5 \%$ variable selon la température et l'épaisseur du revêtement. Les résultats expérimentaux (rapport signal sur bruit et répétabilité notamment) ont cependant montré qu'il est plus judicieux de mesurer la diffusivité thermique d'un revêtement en utilisant la méthode «flash face avant », en particulier lorsque le substrat est épais.

La conductivité thermique du revêtement de $\mathrm{Cr}_{2} \mathrm{O}_{3}$ a également été déterminée entre $23{ }^{\circ} \mathrm{C}$ et $800{ }^{\circ} \mathrm{C}$ à l'aide de la méthode «flash face avant», à partir de mesures simultanées de la diffusivité thermique et de l'effusivité thermique. Les valeurs de la conductivité thermique obtenues ont été comparées à celles évaluées par une méthode de mesure indirecte. Cette comparaison a montré que les deux méthodes donnent des résultats identiques, les valeurs obtenues différant de moins de $7 \%$ sur l'ensemble de la gamme de température et se situant dans la plage d'incertitude de mesure.

Le diffusivimètre du LNE est ainsi opérationnel pour les mesures de la diffusivité thermique et de la conductivité thermique de revêtements épais, entre $23{ }^{\circ} \mathrm{C}$ et $800{ }^{\circ} \mathrm{C}$. Cette installation est utilisée dans deux projets de recherche européens (JRP pour Joint Research Project), financés dans le cadre de l'appel à projet «Energie » de 2009 de l'EMRP (European Metrology Research Programme) d'Euramet, et intitulés “Metrology for improved power plant efficiency" et "Metrology for new generation of nuclear power plants".

Les limitations en termes de durée d'excitation du laser actuellement utilisé pourraient à l'avenir être contournées en changeant le système d'excitation pour un laser d'impulsion plus courte ou des systèmes modulés. Ces systèmes sont compatibles avec le système de détection et ne nécessitent que d'adapter les méthodes de traitement des données.

\section{Remerciements}

Les auteurs sont très reconnaissants au Dr Jean-Claude Krapez de l'Onera pour le développement de la procédure d'estimation des paramètres utilisée.

\section{Références}

[1] DATTA S. et DAS S., "A new high temperature resistant glass-ceramic coating for gas turbine engine components", Bull. Mater. Sci., 28, 7, 2005, 689-696.

[2] ZHU D. et Miller R.A., "Thermal Barrier Coatings for Advanced Gas Turbine and Diesel Engines", Report NASA/TM-1999-209453, National Aeronautics and Space Administration - Glenn Research Center, Cleveland, Ohio, USA, 1999.

[3] BeARDSLEY M.B., "Thick thermal barrier coatings for diesel engines", J. Therm. Spray Tech., 6, 2, 1997, 181186.

[4] Schulz U., Saint-Ramond B., LaVigne O., Moretto P., VANLIESHOUT A. et BöRGER A., "Low thermal conductivity ceramics for turbine blade thermal barrier coating application", Ceramic engineering and science proceedings, 25, 4, 2004, 375-380.

[5] Swab J.J. et P.J. Huang, "Evaluation of Monolithic Ceramics and Ceramic Thermal Barrier Coatings for diesel engines applications", Report ARL-TR-2436, US Army Research Laboratory, Aberdeen, USA, 2001.

[6] SReEkUmar K.P., RAMANathan S., KutTy T.R., SATPUTE R.U. et PADMANABhan P.V., "Novel Ceramic Coatings for Containment of Uranium and Reactive Molten Metals" 16th INSAC Conference, Mumbai, India 15-18 novembre 2005.

[7] Hay B., Filtz J.-R. et Batsale J.-Ch., « Mesure de la diffusivité thermique par la méthode flash », Techniques de l'Ingénieur, R2955, 2004.

[8] PARKer W.J., Jenkins R.J., BUtLeR C.P. et ABbotT G.L., "Flash Method of Determining Thermal Diffusivity, Heat Capacity, and Thermal Conductivity", J. Appl. Phys., 32, 9, 1961, 1679-1684.

[9] Degiovanni A. et Laurent M., « Une nouvelle technique d'identification de la diffusivité thermique par la méthode flash », Rev. Phys. Appl., 21, 1986, 229-237.

[10] Hay B., Rongione L., Filtz J.-R. et Hameury J., "A new reference material for high-temperature thermal 
transport properties - LNE participation in the certification process of Pyroceram 9606", High Temp.-High Pres., 37, 2008, 13-20.

[11] Krapez J.C., Gardette G., Passilly F. et Levesque P., « Participation à la conception d'un banc de mesure de la diffusivité thermique par la méthode flash face avant jusqu'à $800{ }^{\circ} \mathrm{C} »$, Rapport RT 3/03316 DMSE, ONERA, 2002.

[12] Krapez J.-C., Hay B., Demange D., Gardette G., Levesque P. et Passilly F., «Méthode flash en face avant. Optimisation de l'expérience pour un monocouche et un bicouche »- Congrès SFT, Vittel, 2002.

[13] Maillet D., André S., Batsale J.-Ch., Degiovanni A. et MoYne C., "Thermal Quadrupoles. Solving the Heat Equation Through Integral Transforms", Wiley, New York, 2000.

[14] STEHFest H., "Numerical Inversion of Laplace Transforms", Communications of the ACM, 13, 1, 1970, 47-49.

[15] Hay B., Filtz J.-R., Hameury J., DavéE G., Rongione L. et ENOUF O., "Thermal diffusivity measurement of ceramic coatings at high temperature using front-face and rear-face laser flash methods", Int. J. Thermophys., 30, 4, 2009, 1270-1282.

[16] Touloukian Y.S., Powell R.W., Ho C.Y. et Nicolaou M.C., "Thermophysical Properties of Matter. Thermal Diffusivity", vol.10, IFI/Plenum, New York, 1973.

[17] Shanks H.R., Klein A.H. et Danielson G.C., J. Appl. Phys. 38, 1967, 2885.

[18] Filoni L. et Lorenzoni L., High Temp.-High Pres., 23, 1991, 309.

[19] Dobrosavluevic A., Perovic N. et Maglic K., High Temp.-High Pres., 19, 1987, 303.

[20] Hust J.G., "A Fine-Grained Isotropic Graphite for Use as NBS Thermophysical Property RM's from 5 to 2500 K", NBS Special Publication, 1984, 260-289.

[21] BABA T. and CEZAirliyan A., Int. J. Thermophys., 15, 1994, 343.

[22] Hay B., Filtz J.-R., Hameury J. et Rongione L., "Uncertainty of thermal diffusivity measurements by laser flash method", Int. J. Thermophys., 26, 6, 2005, 18831898.

[23] Hay B., Filtz J.-R., Hameury J. et Rongione L., "Uncertainty assessment of thermal diffusivity measurements by flash method - Application to five homogeneous materials", Revue française de métrologie, 14, 2008, 3-11.

[24] DING C., Tong Z. et YAN D., "Properties and applications of plasma-sprayed ceramic coatings", in Plasma SprayingTheory and Applications, (ed.) R. Suryanarayanan, World Scientific Pub. Co., Singapore, 1993, 163-178.
[25] Pang X., GaO K. et Volinsky A., "Microstructure and mechanical properties of chromium oxide coatings", $J$. Mater. Res., 22, 12, 2007, 3531-3537.

[26] Tanaka M., Kitajima Y., Endoh Y., Watanabe M. and NAGITA Y., "Ceramic-metal Composite Coated Piston Ring and Cylinder Liner of Marine Low Speed Diesel Engine", Bull. M.E.S.J., 21, 2, 1993, 77-85.

[27] Richard C.S., Lu J., Béranger G. et Decomps F., "Study of Cr203 Coatings - Part I : Microstructures and Modulus", J. Therm. Spray Tech., 4, 1995, 342-346.

[28] MÄNTYLÄ T., "Thick ceramic coatings”, Euroceram news 7, http://www.euroceram.org/en/news/news7.pdf, 2002 .

[29] PaWlowski L. et FaUchais P., "Thermal transport properties of thermally sprayed coatings", Int. mat. rev., 37 6, 1992, 271-289.

[30] Zhu D., Bansal N.P., LeE K.N. et Miller R.A., "Thermal Conductivity of Ceramic Thermal Barrier and Environmental Barrier Coating Materials", Report NASA/TM-2001-211122, National Aeronautics and Space Administration - Glenn Research Center, Cleveland, Ohio, USA, 2001.

[31] Ding C., HuAng B. et Lin H., "Plasma-sprayed wearresistant ceramic and cermet coating materials", Thin Solid Films, 118, 1984, 485-493.

[32] Vippola M., Vuoristo P., LePistö T. et MäNTYLÄ T., J. Mater. Sci. Lett. 22, 2003, 463.

[33] BRuce R. H. and CANNELl D. S., "Specific heat of $\mathrm{Cr}_{2} \mathrm{O}_{3}$ near the Néel temperature", Phys. rev. B, 15 9, 1977, 44514459.

[34] MARINelli M., Mercuri F., ZAMmit U., Pizzoferrato R., Scudieri F. et DARLADAT D., "Photopyroelectric study of specific heat, thermal conductivity, and thermal diffusivity of $\mathrm{Cr}_{2} \mathrm{O}_{3}$ at the Néel transition", Phys. Rev. B, 49 14, 1994, 9523-9532.

[35] ManN B.S. et PRAKash B., "High temperature friction and wear characteristics of various coarting materials for steam valve spindle application", Wear, 240, 2000, pp. 223-230.

[36] Klemme S., O'NeILl H. et Gmelin E., "The heat capacity of $\mathrm{MgCr}_{2} \mathrm{O}_{4}, \mathrm{FeCr}_{2} \mathrm{O}_{4}$, and $\mathrm{Cr}_{2} \mathrm{O}_{3}$ at low temperatures and derived thermodynamic properties", American Mineralogist, 85, 2000, 1686-1693.

[37] Hay B., Davée G., Filtz J.-R., Hameury J. et RONGIONE L., "Simultaneous measurements of thermal diffusivity and thermal conductivity of ceramic coatings at high temperature by laser flash method", 30th International Thermal Conductivity Conference (ITCC), Pittsburgh, USA, 29 août - 2 septembre 2009. 